\title{
WEIGHTED NORM INEQUALITIES FOR A CLASS OF ROUGH SINGULAR INTEGRALS
}

\author{
H. M. Al-QASSEM
}

Received 11 November 2004 and in revised form 6 February 2005

Weighted norm inequalities are proved for a rough homogeneous singular integral operator and its corresponding maximal truncated singular operator. Our results are essential improvements as well as extensions of some known results on the weighted boundedness of singular integrals.

\section{Introduction}

Throughout this paper, we let $\xi^{\prime}$ denote $\xi /|\xi|$ for $\xi \in \mathbb{R}^{n} \backslash\{0\}$ and $p^{\prime}$ denote the dual exponent to $p$ defined by $1 / p+1 / p^{\prime}=1$. Let $n \geq 2$ and $\mathbf{S}^{n-1}$ represent the unit sphere in $\mathbb{R}^{n}$ equipped with the normalized Lebesgue measure $d \sigma=d \sigma(\cdot)$. Let $K$ be a kernel of Calderon-Zygmund-type on $\mathbb{R}^{n}$ given by

$$
K(x)=\frac{\Omega(x)}{|x|^{n}}
$$

where $\Omega$ is a homogeneous function of degree 0 , integrable over $S^{n-1}$, and satisfies

$$
\int_{S^{n-1}} \Omega(u) d \sigma(u)=0 .
$$

Let $\omega$ be a measurable, almost everywhere positive function on $\mathbb{R}^{n}$. We call such $\omega$ a weight function. We denote by $L^{p}(\omega)$ the $L^{p}(p>0)$ space of all measurable functions $f$ on $\mathbb{R}^{n}$ such that $\|f\|_{L^{p}(\omega)}=\left(\int_{\mathbb{R}^{n}}|f(x)|^{p} \omega(x) d x\right)^{1 / p}<\infty$.

Let $\Gamma(t)$ be a $C^{1}$ function on the interval $\mathbb{R}_{+}$. We define the singular integral operator $T_{\Gamma, \Omega}$ and its maximal truncated singular integral operator $T_{\Gamma, \Omega}^{*}$ by

$$
\begin{aligned}
& T_{\Gamma, \Omega} f(x)=\text { p.v. } \int_{\mathbb{R}^{n}} f\left(x-\Gamma(|y|) y^{\prime}\right) K(y) d y, \\
& T_{\Gamma, \Omega}^{*} f(x)=\sup _{\varepsilon>0}\left|\int_{|y|>\varepsilon} f\left(x-\Gamma(|y|) y^{\prime}\right) K(y) d y\right|,
\end{aligned}
$$

where $y^{\prime}=y /|y| \in \mathbf{S}^{n-1}$, and $f \in \mathscr{S}\left(\mathbb{R}^{n}\right)$, the space of Schwartz functions. 
If $\Gamma(t)=t$, we will denote $T_{\Gamma, \Omega}$ by $T_{\Omega}, T_{\Gamma, \Omega}^{*}$ and by $T_{\Omega}^{*}$.

The investigation of the $L^{p}$ boundedness of $T_{\Omega}$ and $T_{\Omega}^{*}$ was begun by Calderón and Zygmund in [3] and then continued by many authors. In 1956, Calderón and Zygmund showed that the $L^{p}(1<p<\infty)$ boundedness of $T_{\Omega}$ and $T_{\Omega}^{*}$ holds if $\Omega \in L \log ^{+} L\left(\mathbf{S}^{n-1}\right)$ and that this condition is essentially the weakest possible size condition on $\Omega$ for the $L^{p}$ $(1<p<\infty)$ boundedness of $T_{\Omega}$ to hold (see [3]). Some years later, Connett (see [5]) and Coifman and Weiss (see [4]) independently showed that $T_{\Omega}$ is bounded on $L^{p}(1<p<\infty)$ for $\Omega \in H^{1}\left(\mathbf{S}^{n-1}\right)$. Here, $H^{1}\left(\mathbf{S}^{n-1}\right)$ is the Hardy space on the unit sphere which contains the space $L \log ^{+} L\left(\mathbf{S}^{n-1}\right)$ as a proper space. The study of the $L^{p}$ boundedness of the more general class of operators $T_{\Gamma, \Omega}$ and $T_{\Gamma, \Omega}^{*}$ was initiated by Fan and Pan in [8] and continued by many authors. For a sampling of past studies of these operators, see $[1,2,7,8,9]$.

In 1998, Grafakos and Stefanov in [12] introduced the condition

$$
\sup _{\xi \in \mathbf{S}^{n-1}} \int_{\mathbf{S}^{n-1}}|\Omega(y)|\left(\log |\xi \cdot y|^{-1}\right)^{1+\alpha} d \sigma(y)<\infty
$$

and showed that it implies the $L^{p}$ boundedness of $T_{\Omega}$ and $T_{\Omega}^{*}$ for $p$ in a range dependent on the positive exponent $\alpha$. For any $\alpha>0$, let $\mathbf{F}_{\alpha}\left(\mathbf{S}^{n-1}\right)$ denote the family of $\Omega$ 's which are integrable over $\mathrm{S}^{n-1}$ and satisfy (1.4).

Theorem 1.1 (see [12]). Let $\Omega \in \mathbf{F}_{\alpha}\left(\mathbf{S}^{n-1}\right)$ for some $\alpha>0$ and satisfy (1.2). Then

(a) if $\alpha>0, T_{\Omega}$ is bounded on $L^{p}\left(\mathbb{R}^{n}\right)$ for $p \in((2+\alpha) /(1+\alpha), 2+\alpha)$;

(b) if $\alpha>1, T_{\Omega}^{*}$ is bounded on $L^{p}\left(\mathbb{R}^{n}\right)$ for $p \in(1+3 /(1+2 \alpha), 2(2+\alpha) / 3)$.

The range for $p$ was later improved (even for the more general operators $T_{\Gamma, \Omega}$ and $T_{\Gamma, \Omega}^{*}$ and $\Gamma$ is a polynomial) to $((2+2 \alpha) /(1+2 \alpha), 2+2 \alpha)$ (in part (a)) and $((1+2 \alpha) / 2 \alpha, 1+2 \alpha)$ with $\alpha>1 / 2$ (in part (b)) (see [7]). However, it is still unknown whether the latter ranges of indices are sharp. It should be noted that Grafakos and Stefanov in [12] showed that

$$
\begin{aligned}
& \bigcup_{q>1} L^{q}\left(\mathbf{S}^{n-1}\right) \varsubsetneqq \mathbf{F}_{\alpha}\left(\mathbf{S}^{n-1}\right) \quad \text { for any } \alpha>0, \\
& \bigcap_{\alpha>0} \mathbf{F}_{\alpha}\left(\mathbf{S}^{n-1}\right) \nsubseteq H^{1}\left(\mathbf{S}^{n-1}\right) \nsubseteq \bigcup_{\alpha>0} \mathbf{F}_{\alpha}\left(\mathbf{S}^{n-1}\right) .
\end{aligned}
$$

In the meantime, the study of the weighted $L^{p}$ boundedness of $T_{\Omega}$ has also attracted the attention of many authors. For relevant results, one may consult $[6,10,13,14,16$, $17,20]$, among others. We will content ourselves here with recalling only the following pertinent results.

In 1993, Duoandikoetxea [6] proved the following two results.

Theorem 1.2 (see [6]). Suppose that $\Omega \in L^{q}\left(\mathbf{S}^{n-1}\right)$ for some $q>1$. Then $T_{\Omega}$ and $T_{\Omega}^{*}$ are bounded on $L^{p}(\omega)$ if $q^{\prime} \leq p<\infty, p \neq 1$ and $\omega \in A_{p / q^{\prime}}$, where $A_{p}\left(\mathbb{R}^{n}\right)$ denotes the Muckenhoupt class of weights for which the classical Hardy-Littlewood maximal function $M f$ is bounded in $L^{p}(\omega)$. (For the definition and properties of $A_{p}$ weights, see [11].)

For a special class of radial weights $\tilde{A}_{p}\left(\mathbb{R}_{+}\right)$(see the definition in Section 2), Duoandikoetxea proved the following sharper result. 
Theorem 1.3 (see [6]). If $\omega \in \widetilde{A}_{p}\left(\mathbb{R}_{+}\right)$for $1<p<\infty$, then $T_{\Omega}$ is bounded on $L^{p}(\omega)$ provided that $\Omega \in L \log ^{+} L\left(\mathbf{S}^{n-1}\right)$.

Later on, Theorem 1.3 was improved and extended by Fan, Pan and Yang as described in the following theorem.

Theorem 1.4 (see [10]). If $\omega \in \tilde{A}_{p}^{I}\left(\mathbb{R}_{+}\right)$for $1<p<\infty$, then $T_{\Gamma, \Omega}$ and $T_{\Gamma, \Omega}^{*}$ are bounded on $L^{P}(\omega)$ provided that $\Omega \in H^{1}\left(\mathbf{S}^{n-1}\right)$ and $\Gamma$ satisfies either hypothesis $I$ or hypothesis $D$ defined below. Here, $\widetilde{A}_{p}^{I}\left(\mathbb{R}_{+}\right)$is a subclass of $\widetilde{A}_{p}\left(\mathbb{R}_{+}\right)$and its definition will be reviewed in Section 2.

In this paper, we will investigate the weighted $L^{p}(\omega)$ boundedness of the operators $T_{\Gamma, \Omega}$ and $T_{\Gamma, \Omega}^{*}$ for $\omega \in \widetilde{A}_{p}^{I}\left(\mathbb{R}_{+}\right)$and $\Omega \in \mathbf{F}_{\alpha}\left(\mathbf{S}^{n-1}\right)$ for some $\alpha>0$. To state our main results, we need some definitions.

We will need the following definitions from [10].

Definition 1.5. A function $\Gamma$ satisfies "hypothesis I" if

(a) $\Gamma$ is a nonnegative $C^{1}$ function on $(0, \infty)$;

(b) $\Gamma$ is strictly increasing, $\Gamma(2 t) \geq \lambda \Gamma(t)$ for some fixed $\lambda>1$ and $\Gamma(2 t) \leq c \Gamma(t)$ for some constant $c \geq \lambda>1$;

(c) $\Gamma^{\prime}(t) \geq C_{1} \Gamma(t) / t$ on $(0, \infty)$ for some fixed $C_{1} \in\left(0, \log _{2} c\right]$ and $\Gamma^{\prime}(t)$ is monotone on $(0, \infty)$.

Definition 1.6. $\Gamma$ satisfies "hypothesis D" if

(a') $\Gamma$ is a nonnegative $C^{1}$ function on $(0, \infty)$;

(b') $\Gamma$ is strictly decreasing, $\Gamma(t) \geq \lambda \Gamma(2 t)$ for some fixed $\lambda>1$ and $\Gamma(t) \leq c \Gamma(2 t)$ for some constant $c \geq \lambda>1$;

(c') $\left|\Gamma^{\prime}(t)\right| \geq C_{1} \Gamma(t) / t$ on $(0, \infty)$ for some fixed $C_{1} \in\left(0, \log _{2} c\right]$ and $\Gamma^{\prime}(t)$ is monotone on $(0, \infty)$.

Model functions for the $\Gamma$ satisfying hypothesis I are $\Gamma(t)=t^{d}$ with $d>0$, and their linear combinations with positive coefficients. Model functions for the $\Gamma$ satisfying hypothesis $\mathrm{D}$ are $\Gamma(t)=t^{r}$ with $r<0$, and their linear combinations with positive coefficients.

Theorem 1.7. Suppose that $\Omega$ satisfies (1.2) and $\Omega \in \mathbf{F}_{\alpha}\left(\mathbf{S}^{n-1}\right)$ for some $\alpha>0$. Assume that $\Gamma$ satisfies either hypothesis I or hypothesis $D$. If $p \in((2+2 \alpha) /(1+2 \alpha), 2+2 \alpha)$ and $\omega \in \tilde{A}_{p}^{I}\left(\mathbb{R}_{+}\right)$, then the operator $T_{\Gamma, \Omega}$ is bounded on $L^{p}(\omega)$.

Theorem 1.8. Suppose that $\Omega$ satisfies (1.2) and $\Omega \in \mathbf{F}_{\alpha}\left(\mathbf{S}^{n-1}\right)$ for some $\alpha>1 / 2$. Assume that $\Gamma$ satisfies either hypothesis I or hypothesis D. If $p \in((1+2 \alpha) / 2 \alpha, 1+2 \alpha)$ and $\omega \in$ $\tilde{A}_{p}^{I}\left(\mathbb{R}_{+}\right)$, then $T_{\Gamma, \Omega}^{*}$ is bounded on $L^{p}(\omega)$.

Remark 1.9. Obviously, Theorems 1.7 and 1.8 represent an extension of Theorem 1.1 and an improvement and extension over Theorem 1.2 in the case $\omega \in \widetilde{A}_{p}^{I}\left(\mathbb{R}_{+}\right)$because $\Omega$ is allowed to be in the space $\mathbf{F}_{\alpha}\left(\mathbf{S}^{n-1}\right)$ for some $\alpha>0$; and bearing in mind the relation, $L^{d}\left(\mathbf{S}^{n-1}\right) \subset \mathbf{F}_{\alpha}\left(\mathbf{S}^{n-1}\right)$ for all $d>1$.

Throughout this paper, the letter $C$ will stand for a positive constant that may vary at each occurrence. However, $C$ does not depend on any of the essential variables. 


\section{Some definitions and lemmas}

We start this section by reviewing the definition of some special classes of weights and some of their important properties which are relevant to our current study.

Definition 2.1. Let $\omega(t) \geq 0$ and $\omega \in L_{\text {loc }}^{1}\left(\mathbb{R}_{+}\right)$. For $1<p<\infty, \omega \in A_{p}\left(\mathbb{R}_{+}\right)$if there is a positive constant $C$ such that for any interval $I \subset \mathbb{R}_{+}$,

$$
\left(|I|^{-1} \int_{I} \omega(t) d t\right)\left(|I|^{-1} \int_{I} \omega(t)^{-1 /(p-1)} d t\right)^{p-1} \leq C<\infty .
$$

$A_{1}\left(\mathbb{R}_{+}\right)$is the class of weights $\omega$ for which the Hardy-Littlewood maximal operator $M$ satisfies a weak-type estimate in $L^{1}(\omega)$.

It is well known that the class $A_{1}\left(\mathbb{R}_{+}\right)$is also characterized by all weights $\omega$ for which $M \omega(t) \leq C \omega(t)$ for a.e. $t \in \mathbb{R}_{+}$and for some positive constant $C$.

Definition 2.2. Let $1 \leq p<\infty . \omega \in \widetilde{A}_{p}\left(\mathbb{R}_{+}\right)$if $\omega(x)=v_{1}(|x|) \nu_{2}(|x|)^{1-p}$, where either $v_{i} \in$ $A_{1}\left(\mathbb{R}_{+}\right)$is decreasing or $v_{i}^{2} \in A_{1}\left(\mathbb{R}_{+}\right), i=1,2$.

Let $A_{p}^{I}\left(\mathbb{R}^{n}\right)$ be the weight class defined by exchanging the cubes in the definitions of $A_{p}$ for all $n$-dimensional intervals with sides parallel to coordinate axes (see [15]). Let $\tilde{A}_{p}^{I}=\tilde{A}_{p} \cap A_{p}^{I}$. If $\omega \in \tilde{A}_{p}$, it follows from [6] that the Hardy-Littlewood maximal function $M f$ is bounded on $L^{p}\left(\mathbb{R}^{n}, \omega(|x|) d x\right)$. Therefore, if $\omega(t) \in \tilde{A}_{p}\left(\mathbb{R}_{+}\right)$, then $\omega(|x|) \in A_{p}\left(\mathbb{R}^{n}\right)$.

By following the same argument as in the proof of the elementary properties of $A_{p}$ weight class (see, e.g., [11]), we get the following lemma.

LEMma 2.3. If $1 \leq p<\infty$, then the weight class $\tilde{A}_{p}^{I}\left(\mathbb{R}_{+}\right)$has the following properties:

(i) $\widetilde{A}_{p_{1}}^{I} \subset \widetilde{A}_{p_{2}}^{I}$, if $1 \leq p_{1}<p_{2}<\infty$;

(ii) for any $\omega \in \widetilde{A}_{p}^{I}$, there exists an $\varepsilon>0$ such that $\omega^{1+\varepsilon} \in \tilde{A}_{p}^{I}$;

(iii) for any $\omega \in \widetilde{A}_{p}^{I}$ and $p>1$, there exists an $\varepsilon>0$ such that $p-\varepsilon>1$ and $\omega \in \widetilde{A}_{p-\varepsilon}^{I}$.

Definition 2.4. Let $\Gamma(t)$ be a $C^{1}$ function on the interval $\mathbb{R}_{+}$. Define the sequence of measures $\left\{\sigma_{k, \Omega}: k \in \mathbb{Z}\right\}$ and its corresponding maximal operator $\sigma_{\Omega}^{*}$ on $\mathbb{R}^{n}$ by

$$
\begin{gathered}
\int_{\mathbb{R}^{n}} f d \sigma_{k, \Omega}=\int_{2^{k} \leq|y|<2^{k+1}} f\left(\Gamma(|y|) y^{\prime}\right) \frac{\Omega\left(y^{\prime}\right)}{|y|^{n}} d y, \\
\sigma_{\Omega}^{*} f(x)=\sup _{k \in \mathbb{Z}}|| \sigma_{k, \Omega}|* f(x)|,
\end{gathered}
$$

where $\left|\sigma_{k, \Omega}\right|$ is defined in the same way as $\sigma_{k, \Omega}$, but with $\Omega$ replaced by $|\Omega|$.

For $k \in \mathbb{Z}$, set $a_{k}=\Gamma\left(2^{k}\right)$ if $\Gamma$ satisfies hypothesis I and $a_{k}=\left(\Gamma\left(2^{k}\right)\right)^{-1}$ if $\Gamma$ satisfies hypothesis $\mathrm{D}$. Then by the conditions of $\Gamma,\left\{a_{k}\right\}$ is a lacunary sequence of positive numbers with $\inf _{k \in \mathbb{Z}}\left(a_{k+1} / a_{k}\right) \geq \lambda>1$. 
Lemma 2.5. Suppose that $\Omega$ satisfies (1.2) and $\Omega \in \mathbf{F}_{\alpha}\left(\mathbf{S}^{n-1}\right)$ for some $\alpha>0$. Then if $\Gamma$ satisfies hypothesis I,

$$
\begin{gathered}
\| \sigma_{k, \Omega}|| \leq C, \\
\left|\hat{\sigma}_{k, \Omega}(\xi)\right| \leq C\left|a_{k} \xi\right|, \\
\left|\hat{\sigma}_{k, \Omega}(\xi)\right| \leq C|\log | a_{k} \xi||^{-\alpha-1} \text { for }\left|a_{k} \xi\right|>e,
\end{gathered}
$$

and if $\Gamma$ satisfies hypothesis D,

$$
\begin{gathered}
\| \sigma_{k, \Omega}|| \leq C, \\
\left|\hat{\sigma}_{k, \Omega}(\xi)\right| \leq C\left|a_{k}^{-1} \xi\right|, \\
\left|\hat{\sigma}_{k, \Omega}(\xi)\right| \leq C|\log | a_{k}^{-1} \xi||^{-\alpha-1} \text { for }\left|a_{k}^{-1} \xi\right|>e,
\end{gathered}
$$

for some positive constant $C$ independent of $k$ and $\xi$, where $\left\|\sigma_{k, \Omega}\right\|$ stands for the total variation of $\sigma_{k, \Omega}$.

Proof. We will only present the proof of the lemma if $\Gamma$ satisfies hypothesis $I$, since the proof for the case that $\Gamma$ satisfies hypothesis $D$ will be essentially the same. It is easy to verify that (2.3) holds for some positive constant $C$. By a change of variable, we have

$$
\widehat{\sigma}_{k, \Omega}(\xi)=\int_{S^{n-1}} \int_{1}^{2} e^{-i \Gamma\left(2^{k} t\right) \xi \cdot x} \Omega(x) \frac{d t}{t} d \sigma(x) .
$$

By (2.7) and the mean zero property (1.2) of $\Omega$, we get

$$
\left|\hat{\sigma}_{k, \Omega}(\xi)\right| \leq \int_{S^{n-1}} \int_{1}^{2}\left|e^{-i \Gamma\left(2^{k} t\right) \xi \cdot x}-1\right||\Omega(x)| \frac{d t}{t} d \sigma(x) .
$$

which easily yields the estimate in (2.4).

Now, we turn to the proof of (2.5). Again, by (2.7), we have

$$
\left|\widehat{\sigma}_{k, \Omega}(\xi)\right| \leq \int_{S^{n-1}}\left|I_{k}(t, x, \xi)\right||\Omega(x)| d \sigma(x),
$$

where

$$
I_{k}(t, x, \xi)=\int_{1}^{2} e^{-i \Gamma\left(2^{k} t\right) \xi \cdot x} \frac{d t}{t}
$$

We notice that

$$
I_{k}(t, x, \xi)=\int_{1}^{2} H^{\prime}(t) \frac{d t}{t}, \quad H(t)=\int_{1}^{t} e^{-i \Gamma\left(2^{k} s\right) \xi \cdot x} d s, \quad 1 \leq s \leq 2 .
$$

Now, using the assumptions on $\Gamma$, we obtain

$$
\frac{d}{d s}\left(\Gamma\left(2^{k} s\right)\right)=2^{k} \Gamma^{\prime}\left(2^{k} s\right) \geq C_{1} \frac{\Gamma\left(2^{k} s\right)}{s} \geq C_{1} \frac{\Gamma\left(2^{k}\right)}{t} \quad \text { for } 1 \leq s \leq t \leq 2 .
$$


By applying van der Corput's lemma (see [18]), $|H(t)| \leq C_{1}^{-1}\left|\Gamma\left(2^{k}\right) \xi\right|^{-1}\left|\xi^{\prime} \cdot x\right|^{-1} t$, for $1 \leq t \leq 2$. Thus by integrating by parts, we have

$$
\left|I_{k}(t, x, \xi)\right| \leq C\left|\Gamma\left(2^{k}\right) \xi\right|^{-1}\left|\xi^{\prime} \cdot x\right|^{-1} .
$$

Now combining this bound with the trivial bound $\left|I_{k}(t, x, \xi)\right| \leq(\log 2)$ and using the fact that $t^{\alpha+1} / \log t$ is increasing on $(e, \infty)$, we get

$$
\left|I_{k}(t, x, \xi)\right| \leq C \frac{\left(\log \left((3 / 2)\left|\xi^{\prime} \cdot x\right|^{-1}\right)\right)^{\alpha+1}}{\left(\log \left|\Gamma\left(2^{k}\right) \xi\right|\right)^{\alpha+1}} \quad \text { if }\left|\Gamma\left(2^{k}\right) \xi\right|>e .
$$

Therefore, by (2.7), (2.9), and the assumption on $\Omega$, we obtain (2.5). This completes the proof of the lemma.

Lemma 2.6. Let $\omega \in \widetilde{A}_{p}\left(\mathbb{R}_{+}\right)$and $\Omega \in L^{1}\left(\mathbf{S}^{n-1}\right)$. Assume that $\Gamma$ satisfies either hypothesis I or hypothesis $D$. Then

$$
\left\|\sigma_{\Omega}^{*}(f)\right\|_{L^{p}(\omega)} \leq C_{p}\|\Omega\|_{L^{1}\left(\mathbf{S}^{n-1}\right)}\|f\|_{L^{p}(\omega)}
$$

for $1<p<\infty$, where $C_{p}$ is independent of $f$.

Proof. By definition of $\sigma_{k, \Omega}$, we have

$$
\begin{aligned}
|| \sigma_{k, \Omega}|* f(x)| & \leq\left(\int_{2^{k}}^{2^{k+1}} \int_{S^{n-1}}\left|\Omega\left(y^{\prime}\right)\right|\left|f\left(x-\Gamma(t) y^{\prime}\right)\right| d \sigma\left(y^{\prime}\right) \frac{d t}{t}\right) \\
& \leq C\left(\int_{S^{n-1}}\left|\Omega\left(y^{\prime}\right)\right| M_{\Gamma, y^{\prime}}(|f|)(x) d \sigma\left(y^{\prime}\right)\right),
\end{aligned}
$$

where

$$
M_{\Gamma, y^{\prime}} f(x)=\sup _{k \in \mathbb{Z}}\left|\int_{2^{k}}^{2^{k+1}} f\left(x-\Gamma(t) y^{\prime}\right) \frac{d t}{t}\right| .
$$

Let $s=\Gamma(t)$. Assume first that $\Gamma$ satisfies hypothesis I. By the assumptions on $\Gamma$, we have $d t / t \leq d s / C_{1} s$. So, by a change of variable, we have

$$
\begin{aligned}
\mathcal{M}_{\Gamma, y^{\prime}} f(x) & \leq \frac{1}{C_{1}} \sup _{k \in \mathbb{Z}}\left(\int_{\Gamma\left(2^{k}\right)}^{\Gamma\left(2^{k+1}\right)}\left|f\left(x-s y^{\prime}\right)\right| \frac{d s}{s}\right) \\
& \leq \frac{1}{C_{1}} \sup _{r>0}\left(\int_{\Gamma(r / 2)}^{\Gamma(r)}\left|f\left(x-s y^{\prime}\right)\right| \frac{d s}{s}\right) .
\end{aligned}
$$

Now, by condition (a) or condition (b), we have

$$
\begin{aligned}
\mathcal{M}_{\Gamma, y^{\prime}} f(x) & \leq \frac{1}{C_{1}} \sup _{r>0}\left(\int_{\Gamma(r / 2)}^{c \Gamma(r / 2)}\left|f\left(x-s y^{\prime}\right)\right| \frac{d s}{s}\right) \\
& \leq C M_{y^{\prime}} f(x),
\end{aligned}
$$


where

$$
M_{y^{\prime}} f(x)=\sup _{R>0} R^{-1} \int_{0}^{R}\left|f\left(x-s y^{\prime}\right)\right| d s
$$

is the Hardy-Littlewood maximal function of $f$ in the direction of $y^{\prime}$. On the other hand, if $\Gamma$ satisfies hypothesis $\mathrm{D}$, we use a similar argument as above to get $d t / t \leq-d s / C_{1} s$ and

$$
\begin{aligned}
\mathcal{M}_{\Gamma, y^{\prime}} f(x) & \leq \frac{1}{C_{1}} \sup _{r>0}\left(\int_{\Gamma(r)}^{\Gamma(r / 2)}\left|f\left(x-s y^{\prime}\right)\right| \frac{d s}{s}\right) \\
& \leq \frac{1}{C_{1}} \sup _{r>0}\left(\int_{(1 / c) \Gamma(r / 2)}^{\Gamma(r / 2)}\left|f\left(x-s y^{\prime}\right)\right| \frac{d s}{s}\right) \\
& \leq C M_{y^{\prime}} f(x) .
\end{aligned}
$$

So in either case, by (2.19)-(2.21) and Minkowski's inequality for integrals, we get

$$
\left\|\sigma_{\Omega}^{*} f\right\|_{L^{p}(\omega)} \leq C\left(\int_{S^{n-1}}\left|\Omega\left(y^{\prime}\right)\right|\left\|M_{y^{\prime}}(|f|)\right\|_{L^{p}(\omega)} d \sigma\left(y^{\prime}\right)\right)
$$

By $[6,(8)]$ and since $\omega \in \tilde{A}_{p}\left(\mathbb{R}_{+}\right)$, we have

$$
\left\|M_{y^{\prime}} f\right\|_{L^{p}(\omega)} \leq C\|f\|_{L^{p}(\omega)}
$$

with $C$ independent of $y^{\prime}$. By (2.22) and (2.23), we get (2.15) which finishes the proof of the lemma.

Lemma 2.7. Let $1<p<\infty$ and $\omega \in \widetilde{A}_{p}\left(\mathbb{R}_{+}\right)$. Then there exists a positive constant $C_{p}$ such that the inequality

$$
\left\|\left(\sum_{k \in \mathbb{Z}}|| \sigma_{k, \Omega}\left|* g_{k}\right|^{2}\right)^{1 / 2}\right\|\left\|_{L^{p}(\omega)} \leq C_{p}\right\|\left(\sum_{k \in \mathbb{Z}}\left|g_{k}\right|^{2}\right)^{1 / 2} \|_{L^{p}(\omega)}
$$

holds for any sequence of functions $\left\{g_{k}\right\}_{k \in \mathbb{Z}}$ on $\mathbb{R}^{n}$.

Proof. By Lemma 2.6, we get

$$
\left\|\sup _{k \in \mathbb{Z}}|| \sigma_{k, \Omega}\left|* g_{k}\right|\right\|_{L^{p}(\omega)} \leq\left\|\sigma_{\Omega}^{*}\left(\sup _{k \in \mathbb{Z}}\left|g_{k}\right|\right)\right\|_{L^{p}(\omega)} \leq C\left\|\left(\sup _{k \in \mathbb{Z}}\left|g_{k}\right|\right)\right\|_{L^{p}(\omega)}
$$

On the other hand, there exists a nonnegative function $f$ in $L^{p^{\prime}}(\omega)$ with $\|f\|_{L^{p^{\prime}}(\omega)} \leq 1$ such that

$$
\left\|\sum_{k \in \mathbb{Z}}|| \sigma_{k, \Omega}\left|* g_{k}\right|\right\|_{L^{p}(\omega)}=\int_{\mathbb{R}^{n}} \sum_{k \in \mathbb{Z}}|| \sigma_{k, \Omega}\left|* g_{k}(x)\right| \omega(x) f(x) d x .
$$


Thus, by Fubini's theorem and Hölder's inequality, we get

$$
\begin{aligned}
\left\|\sum_{k \in \mathbb{Z}}|| \sigma_{k, \Omega}\left|* g_{k}\right|\right\|_{L^{p}(\omega)} & \leq \int_{\mathbb{R}^{n}} \sum_{k \in \mathbb{Z}}\left|g_{k}(x)\right| \sigma_{\Omega}^{*}((\widetilde{\omega f}))(-x) d x \\
& \left.\leq\left\|\sum_{k \in \mathbb{Z}}\left|g_{k}\right|\right\|_{L^{p}(\omega)} \| \sigma_{\Omega}^{*}(\widetilde{(\omega f})\right) \|_{L^{p^{\prime}}\left(\omega^{1-p^{\prime}}\right)},
\end{aligned}
$$

where $\tilde{u}(x)=u(-x)$. Since $\omega \in \widetilde{A}_{p}\left(\mathbb{R}_{+}\right)$if and only if $\omega^{1-p^{\prime}} \in \widetilde{A}_{p^{\prime}}\left(\mathbb{R}_{+}\right)$, by Lemma 2.6, we get

$$
\left\|\sum_{k \in \mathbb{Z}}|| \sigma_{k, \Omega}\left|* g_{k}\right|\right\|_{L^{p}(\omega)} \leq C_{p}\left\|\sum_{k \in \mathbb{Z}}\left|g_{k}\right|\right\|_{L^{p}(\omega)} .
$$

Therefore, we can interpolate (2.25) and (2.28) (see [11, page 481] for the vector-valued interpolation) to get (2.24). The lemma is proved.

\section{Proof of Theorems 1.7 and 1.8}

Proof of Theorem 1.7. We will present the proof of Theorem 1.7 only for the case where $\Gamma$ satisfies hypothesis $\mathrm{I}$, since the proof for the case where $\Gamma$ satisfies hypothesis $\mathrm{D}$ is essentially the same. Let $\left\{\psi_{k}\right\}_{-\infty}^{\infty}$ be a smooth partition of unity in $(0, \infty)$ adapted to the intervals $I_{k}=\left[\left(a_{k+1}\right)^{-1},\left(a_{k-1}\right)^{-1}\right]$. To be precise, we require the following:

$$
\begin{gathered}
\psi_{k} \in C^{\infty}, \quad 0 \leq \psi_{k} \leq 1, \quad \sum_{k}\left(\psi_{k}(t)\right)^{2}=1, \\
\operatorname{supp} \psi_{k} \subseteq I_{k}, \quad\left|\frac{d^{s} \psi_{k}(t)}{d t^{s}}\right| \leq \frac{C_{s}}{t^{s}},
\end{gathered}
$$

where $C^{\infty}$ denotes the class of all infinitely differentiable functions on $(0, \infty)$ and $C_{s}$ is independent of the lacunary sequence $\left\{\Gamma\left(2^{k}\right): k \in \mathbb{Z}\right\}$. (We remark at this point that if $\Gamma$ satisfies hypothesis $\mathrm{D}$, the partition of unity needed in our proof should have the same properties as above except that we need it to be adapted to the intervals $\left.\left[a_{k-1}, a_{k+1}\right]\right)$. Define the multiplier operators $S_{k}$ in $\mathbb{R}^{n}$ by

$$
\left(\widehat{S_{k} f}\right)(\xi)=\psi_{k}(|\xi|) \widehat{f}(\xi) .
$$

Define

$$
Q_{j}(f)=\sum_{k \in \mathbb{Z}} S_{k+j}\left(\sigma_{k, \Omega} * S_{k+j} f\right) .
$$

Now

$$
T_{\Gamma, \Omega}(f)=\sum_{k \in \mathbb{Z}} \sigma_{k, \Omega} * f=\sum_{k \in \mathbb{Z}} \sigma_{k, \Omega} *\left(\sum_{j \in \mathbb{Z}} S_{k+j} S_{k+j} f\right)=\sum_{j \in \mathbb{Z}} Q_{j}(f)
$$


holds at least for $f \in \mathscr{S}\left(\mathbb{R}^{n}\right)$. By Plancherel's theorem, we have

$$
\left\|Q_{j}(f)\right\|_{L^{2}}^{2} \leq \sum_{k \in \mathbb{Z}} \int_{\mathbb{R}^{n}}|\hat{f}(\xi)|^{2} \chi_{\Delta_{k+j}}(\xi)\left|\hat{\sigma}_{k, \Omega}(\xi)\right|^{2} d \xi,
$$

where

$$
\Delta_{k}=\left\{\xi \in \mathbb{R}^{n}:\left(a_{k+1}\right)^{-1} \leq|\xi|<\left(a_{k-1}\right)^{-1}\right\}
$$

By a straightforward computation and (2.3)-(2.5), we get

$$
\begin{aligned}
& \left\|Q_{j}(f)\right\|_{L^{2}} \leq C|j|^{-\alpha-1}\|f\|_{L^{2}} \quad \text { if } j \leq-1, \\
& \left\|Q_{j}(f)\right\|_{L^{2}} \leq C \lambda^{-j}\|f\|_{L^{2}} \quad \text { if } j \geq 0
\end{aligned}
$$

and hence,

$$
\left\|Q_{j}(f)\right\|_{L^{2}} \leq C(1+|j|)^{-\alpha-1}\|f\|_{L^{2}} \quad \forall j \in \mathbb{Z} .
$$

On the other hand, for every $p \in(1, \infty)$ and $\omega \in \widetilde{A}_{p}^{I}\left(\mathbb{R}_{+}\right)$,

$$
\begin{aligned}
\left\|Q_{j}(f)\right\|_{L^{p}(\omega)} & \leq C_{p}\left\|\left(\sum_{k \in \mathbb{Z}}\left|\sigma_{k, \Omega} * S_{k+j} f\right|^{2}\right)^{1 / 2}\right\|_{L^{p}(\omega)} \\
& \leq C_{p}\left\|\left(\sum_{k \in \mathbb{Z}}\left|S_{k+j} f\right|^{2}\right)^{1 / 2}\right\| \|_{L^{p}(\omega)} \\
& \leq C_{p}\|f\|_{L^{p}(\omega)}
\end{aligned}
$$

where the first and the last inequalities follow by the weighted Littlewood-Paley theory since $\omega \in \tilde{A}_{p}^{I}\left(\mathbb{R}_{+}\right) \subset \tilde{A}_{p}\left(\mathbb{R}_{+}\right) \subset A_{p}\left(\mathbb{R}_{+}\right)$, whereas the second inequality follows from Lemma 2.7. Thus, by Lemma 2.3, there is an $\varepsilon>0$ such that

$$
\left\|Q_{j}(f)\right\|_{L^{p}\left(\omega^{1+\varepsilon}\right)} \leq C_{p}\|f\|_{L^{p}\left(\omega^{1+\varepsilon}\right)}
$$

for every $\omega \in \tilde{A}_{p}^{I}\left(\mathbb{R}_{+}\right)$and $1<p<\infty$. By interpolating between (3.8) and (3.9) with $\omega=1$, for every $p \in((2+2 \alpha) /(1+2 \alpha), 2+2 \alpha)$, there is a $\theta_{p}>1$ such that

$$
\left\|Q_{j}(f)\right\|_{L^{p}} \leq C(1+|j|)^{-\theta_{p}}\|f\|_{L^{p}}
$$

holds for $j \in \mathbb{Z}$. Using Stein and Weiss' interpolation theorem with change of measure, we interpolate (3.10) with (3.11) to get that, for every $p \in((2+2 \alpha) /(1+2 \alpha), 2+2 \alpha)$ and $\omega \in \tilde{A}_{p}^{I}\left(\mathbb{R}_{+}\right)$, there is an $\eta_{p}>1$ such that

$$
\left\|Q_{j}(f)\right\|_{L^{p}(\omega)} \leq C(1+|j|)^{-\eta_{p}}\|f\|_{L^{p}(\omega)}
$$


holds for $j \in \mathbb{Z}$. Therefore, by (3.4) and (3.12), we get

$$
\left\|T_{\Gamma, \Omega}(f)\right\|_{L^{p}(\omega)} \leq \sum_{j \in \mathbb{Z}}\left\|Q_{j}(f)\right\|_{L^{p}(\omega)} \leq C\|f\|_{L^{p}(\omega)}
$$

for every $p \in((2+2 \alpha) /(1+2 \alpha), 2+2 \alpha)$ and $\omega \in \widetilde{A}_{p}^{I}\left(\mathbb{R}_{+}\right)$. This completes the proof of Theorem 1.7.

Proof of Theorem 1.8. Assume that $p \in((1+2 \alpha) / 2 \alpha, 1+2 \alpha)$ and $\omega \in \tilde{A}_{p}^{I}\left(\mathbb{R}_{+}\right)$. For any $\varepsilon>$ 0 , there is an integer $k$ such that $a_{k} \leq \varepsilon<a_{k+1}$. So we have

$$
T_{\Gamma, \Omega}^{*}(f) \leq \sigma_{\Omega}^{*}(f)+H(f)
$$

where $H(f)=\sup _{k \in \mathbb{Z}}\left|T_{k}(f)\right|$ and $T_{k}(f)=\sum_{j=k}^{\infty} \sigma_{j, \Omega} * f$. By (3.14) and Lemma 2.6, we notice that the proof of Theorem 1.8 is completed if we can show that

$$
\|H(f)\|_{L^{p}(\omega)} \leq C_{p}\|f\|_{L^{p}(\omega)}
$$

for $p \in((1+2 \alpha) / 2 \alpha, 1+2 \alpha)$ and $\omega \in \widetilde{A}_{p}^{I}\left(\mathbb{R}_{+}\right)$. So, we turn to the proof of (3.15). Let $\varphi \in \mathscr{S}\left(\mathbb{R}^{n}\right)$ be such that $\varphi(\xi)=1$ for $|\xi|<1 / \lambda$ and $\varphi(\xi)=0$ for $|\xi|>\lambda$. Define $\phi, \phi_{k}$ by $(\hat{\phi})=\varphi$ and $\phi_{k}(\xi)=\left(1 /\left(a_{k}\right)^{n}\right) \phi_{k}\left(\xi / a_{k}\right)$. Decompose $T_{k}(f)$ as

$$
\begin{aligned}
T_{k}(f) & =\left(\delta-\phi_{k}\right) * \sum_{j=k}^{\infty} \sigma_{j, \Omega} * f+\phi_{k} * T_{\Gamma, \Omega}(f)-\phi_{k} * \sum_{j=-\infty}^{k-1} \sigma_{j, \Omega} * f \\
& =T_{k}^{(1)} f+T_{k}^{(2)} f+T_{k}^{(3)} f,
\end{aligned}
$$

where $\delta$ is the Dirac delta function. By Theorem 1.7 and since $\omega \in \tilde{A}_{p}\left(\mathbb{R}_{+}\right) \subset A_{p}\left(\mathbb{R}_{+}\right)$, we immediately get

$$
\left\|\sup _{k \in \mathbb{Z}}\left|T_{k}^{(2)} f\right|\right\|_{L^{p}(\omega)} \leq C\left\|M\left(T_{\Gamma, \Omega}\right)\right\|_{L^{p}(\omega)} \leq C_{p}\|f\|_{L^{p}(\omega)}
$$

for $p \in((1+2 \alpha) / 2 \alpha, 1+2 \alpha)$ and $\omega \in \tilde{A}_{p}^{I}\left(\mathbb{R}_{+}\right)$. By definition of $T_{k}^{(3)} f$, we get

$$
\sup _{k \in \mathbb{Z}}\left|T_{k}^{(3)} f\right| \leq \sum_{j=1}^{\infty} \sup _{k \in \mathbb{Z}}\left|\sigma_{k-j, \Omega} * \phi_{k} * f(x)\right|=\sum_{j=1}^{\infty} U_{j}(f) .
$$

By Lemma 2.6, we have

$$
\left\|U_{j}(f)\right\|_{L^{p}(\omega)} \leq\left\|M\left(\sigma_{\Omega}^{*}(f)\right)\right\|_{L^{p}(\omega)} \leq C_{p}\|f\|_{L^{p}(\omega)}
$$

for every $\omega \in \tilde{A}_{p}^{I}\left(\mathbb{R}_{+}\right)$and $1<p<\infty$. Thus, by Lemma 2.3 , there is an $\varepsilon>0$ such that

$$
\left\|U_{j}(f)\right\|_{L^{p}\left(\omega^{1+\varepsilon}\right)} \leq C_{p}\|f\|_{L^{p}\left(\omega^{1+\varepsilon}\right)}
$$


for every $\omega \in \tilde{A}_{p}^{I}\left(\mathbb{R}_{+}\right)$and $1<p<\infty$. On the other hand,

$$
U_{j}(f) \leq\left(\sum_{k \in \mathbb{Z}}\left|\sigma_{k-j, \Omega} * \phi_{k} * f\right|^{2}\right)^{1 / 2}
$$

Thus, by Plancherel's theorem and Lemma 2.5, we have

$$
\begin{aligned}
\left\|U_{j}(f)\right\|_{2}^{2} & \leq \int_{\mathbb{R}^{n}} \sum_{k \in \mathbb{Z}}\left|\hat{\sigma}_{k-j, \Omega}(\xi)\right|^{2}\left|\varphi_{k}(\xi)\right|^{2}|\hat{f}(\xi)|^{2} d \xi \\
& \leq \int_{\mathbb{R}^{n}} \sum_{\left|a_{k} \xi\right| \leq \lambda}\left|a_{k-j} \xi\right|^{2}|\hat{f}(\xi)|^{2} d \xi \\
& \leq C \lambda^{-2 j}\|f\|_{2}^{2} .
\end{aligned}
$$

Thus, by interpolating between (3.22) and (3.20) (with $\omega=1$ ), we get

$$
\left\|U_{j}(f)\right\|_{p} \leq C \lambda^{-j \beta(p)}\|f\|_{p}
$$

for $1<p<\infty$ and for some $\beta(p)>0$. Now, using Stein and Weiss' interpolation theorem with change of measures [19], we may interpolate between (3.20) and (3.23) to obtain a positive number $v_{p}$ such that

$$
\left\|U_{j}(f)\right\|_{L^{p}(\omega)} \leq C \lambda^{-j v_{p}}\|f\|_{L^{p}(\omega)}
$$

which implies

$$
\left\|\sup _{k \in \mathbb{Z}}\left|T_{k}^{(3)} f\right|\right\|_{L^{p}(\omega)} \leq C_{p}\|f\|_{L^{p}(\omega)}
$$

for every $\omega \in \tilde{A}_{p}^{I}\left(\mathbb{R}_{+}\right)$and $1<p<\infty$.

Finally, we will prove that

$$
\left\|\sup _{k \in \mathbb{Z}}\left|T_{k}^{(1)} f\right|\right\|_{L^{p}(\omega)} \leq C_{p}\|f\|_{L^{p}(\omega)}
$$

for $p \in((1+2 \alpha) / 2 \alpha, 1+2 \alpha)$ and $\omega \in \tilde{A}_{p}^{I}\left(\mathbb{R}_{+}\right)$. The proof of this inequality is similar to the proof of (3.25). In fact, we have

$$
\sup _{k \in \mathbb{Z}}\left|T_{k}^{(1)} f\right| \leq \sum_{j=1}^{\infty}\left(\sup _{k \in \mathbb{Z}}\left|\sigma_{k+j, \Omega} *\left(\delta-\phi_{k}\right) * f(x)\right|\right)=\sum_{j=1}^{\infty} \Upsilon_{j}(f) .
$$

By Lemma 2.6 and the $L^{p}(\omega)$ boundedness of $M$, we get

$$
\left\|\Upsilon_{j}(f)\right\|_{L^{p}(\omega)} \leq C_{p}\|f\|_{L^{p}(\omega)}
$$


for every $\omega \in \tilde{A}_{p}^{I}\left(\mathbb{R}_{+}\right)$and $1<p<\infty$. Also, by Plancherel's theorem,

$$
\begin{aligned}
\left\|\Upsilon_{j}(f)\right\|_{2}^{2} & \leq \int_{\mathbb{R}^{n}} \sum_{k \in \mathbb{Z}}\left|\hat{\sigma}_{k+j, \Omega}(\xi)\right|^{2}\left|1-\varphi_{k}(\xi)\right|^{2}|\hat{f}(\xi)|^{2} d \xi \\
& =\sum_{k \in \mathbb{Z}} \int_{1 / \lambda \leq\left|a_{k} \xi\right|}\left|\hat{\sigma}_{k+j, \Omega}(\xi)\right|^{2}|\hat{f}(\xi)|^{2} d \xi \\
& \leq \sum_{k=-\infty}^{\infty} \sum_{s=0}^{\infty} \int_{\lambda^{s} \leq\left|\lambda a_{k} \xi\right|<\lambda^{s+1}}|\log | a_{k+j} \xi||^{-2 \alpha-2}|\hat{f}(\xi)|^{2} d \xi \\
& \leq \sum_{k=-\infty}^{\infty} \sum_{s=0}^{\infty}\left(\frac{1}{j+s-1}\right)^{2 \alpha+2} \int_{\lambda^{s} \leq\left|\lambda a_{k} \xi\right|<\lambda^{s+1}}|\hat{f}(\xi)|^{2} d \xi \\
& \leq \sum_{s=0}^{\infty}\left(\frac{1}{j+s-1}\right)^{2 \alpha+2}\|f\|_{2}^{2} \\
& \leq C j^{-1-2 \alpha}\|f\| 1_{2}^{2} .
\end{aligned}
$$

Thus Theorem 1.8 is proved.

We end this section with the following result concerning power weights $|x|^{\gamma}$. One of the important special classes of radial weights is the power weights $|x|^{\gamma}, \gamma \in \mathbb{R}$. It is known that $|x|^{\gamma} \in A_{p}\left(\mathbb{R}^{n}\right)$ if and only if $-n<\gamma<n(p-1)$.

Our result regarding this class of weights is the following.

Theorem 3.1. Let $1<p<\infty$. Assume that $\Gamma$ satisfies either hypothesis I or hypothesis D. If $\Omega \in \mathbf{F}_{\alpha}\left(\mathbf{S}^{n-1}\right)$ for some $\alpha>0$ and satisfies (1.2), then for $\omega(x)=|x|^{\gamma}$ with $\gamma \in(-1, p-1)$, $T_{\Gamma, \Omega}$ is bounded on $L^{p}(\omega)$ for $p \in((2+2 \alpha) /(1+2 \alpha), 2+2 \alpha)$ and $T_{\Gamma, \Omega}^{*}$ is bounded on $L^{p}(\omega)$ for $p \in((1+2 \alpha) / 2 \alpha, 1+2 \alpha)$.

A proof of this theorem can be obtained by applying the results in Theorems 1.7 and 1.8 and noticing that $|x|^{\gamma} \in \widetilde{A}_{p}^{I}\left(\mathbb{R}_{+}\right)$for $\gamma \in(-1, p-1)$.

\section{Acknowledgment}

The author would like to thank the referees for their valuable comments and suggestions.

\section{References}

[1] A. J. Al-Hasan and D. Fan, $L^{p}$-boundedness of a singular integral operator, Canad. Math. Bull. 41 (1998), no. 4, 404-412.

[2] H. Al-Qassem and A. Al-Salman, $L^{p}$ boundedness of a class of singular integral operators with rough kernels, Turkish J. Math. 25 (2001), no. 4, 519-533.

[3] A. P. Calderón and A. Zygmund, On singular integrals, Amer. J. Math. 78 (1956), 289-309.

[4] R. R. Coifman and G. Weiss, Extensions of Hardy spaces and their use in analysis, Bull. Amer. Math. Soc. 83 (1977), no. 4, 569-645.

[5] W. C. Connett, Singular integrals near $L^{1}$, Harmonic Analysis in Euclidean Spaces, Proc. Sympos. Pure Math., vol. 35, Amer. Math. Soc., Rhode Island, 1979, pp. 163-165.

[6] J. Duoandikoetxea, Weighted norm inequalities for homogeneous singular integrals, Trans. Amer. Math. Soc. 336 (1993), no. 2, 869-880. 
[7] D. Fan, K. Guo, and Y. Pan, A note of a rough singular integral operator, Math. Inequal. Appl. 2 (1999), no. 1, 73-81.

[8] D. Fan and Y. Pan, $L^{2}$ boundedness of a singular integral operator, Publ. Mat. 41 (1997), 317-333.

[9] - A singular integral operator with rough kernel, Proc. Amer. Math. Soc. 125 (1997), no. 12, 3695-3703.

[10] D. Fan, Y. Pan, and D. Yang, A weighted norm inequality for rough singular integrals, Tohoku Math. J. (2) 51 (1999), no. 2, 141-161.

[11] J. García-Cuerva and J. L. Rubio de Francia, Weighted Norm Inequalities and Related Topics, North-Holland Mathematics Studies, vol. 116, North-Holland Publishing, Amsterdam, 1985.

[12] L. Grafakos and A. Stefanov, $L^{p}$ bounds for singular integrals and maximal singular integrals with rough kernels, Indiana Univ. Math. J. 47 (1998), no. 2, 455-469.

[13] J. Álvarez, R. J. Bagby, D. S. Kurtz, and C. Pérez, Weighted estimates for commutators of linear operators, Studia Math. 104 (1993), no. 2, 195-209.

[14] S. Hofmann, Weighted inequalities for commutators of rough singular integrals, Indiana Univ. Math. J. 39 (1990), no. 4, 1275-1304.

[15] D. S. Kurtz, Littlewood-Paley and multiplier theorems on weighted $L^{p}$ spaces, Trans. Amer. Math. Soc. 259 (1980), no. 1, 235-254.

[16] D. S. Kurtz and R. L. Wheeden, A note on a singular integral with weights, Trans. Amer. Math. Soc. 81 (1993), 391-397.

[17] B. Muckenhoupt and R. L. Wheeden, Weighted norm inequalities for singular and fractional integrals, Trans. Amer. Math. Soc. 161 (1971), 249-258.

[18] E. M. Stein, Harmonic Analysis: Real-Variable Methods, Orthogonality, and Oscillatory Integrals, Princeton Mathematical Series, vol. 43, Princeton University Press, New Jersey, 1993.

[19] E. M. Stein and G. Weiss, Interpolation of operators with change of measures, Trans. Amer. Math. Soc. 87 (1958), 159-172.

[20] D. K. Watson, Weighted estimates for singular integrals via Fourier transform estimates, Duke Math. J. 60 (1990), no. 2, 389-399.

H. M. Al-Qassem: Department of Mathematics, Faculty of Science, Yarmouk University, Irbid, Jordan

E-mail address: husseink@yu.edu.jo 


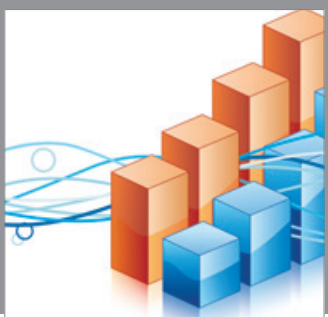

Advances in

Operations Research

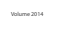

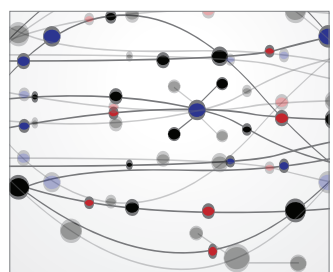

\section{The Scientific} World Journal
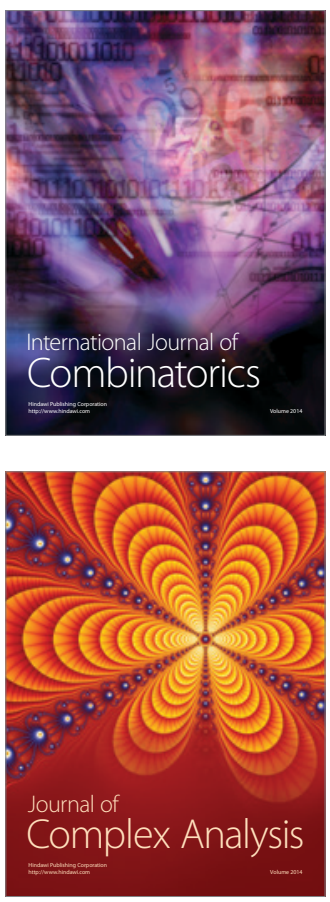

International Journal of

Mathematics and

Mathematical

Sciences
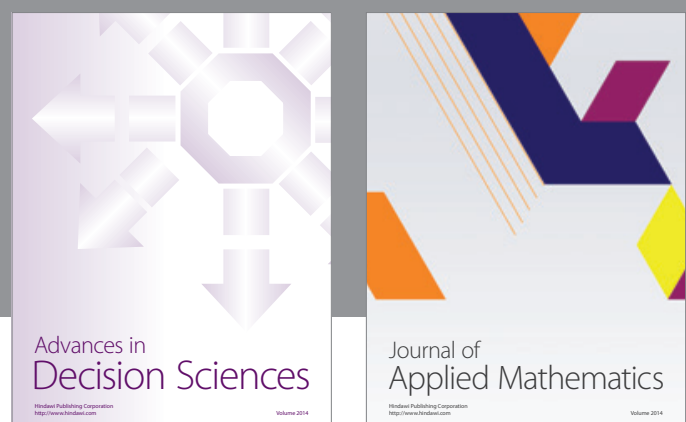

Journal of

Applied Mathematics
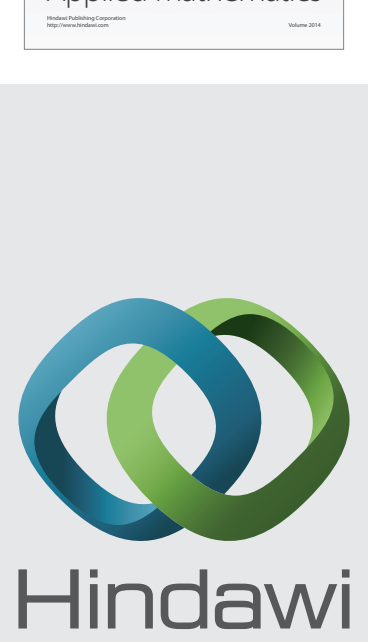

Submit your manuscripts at http://www.hindawi.com
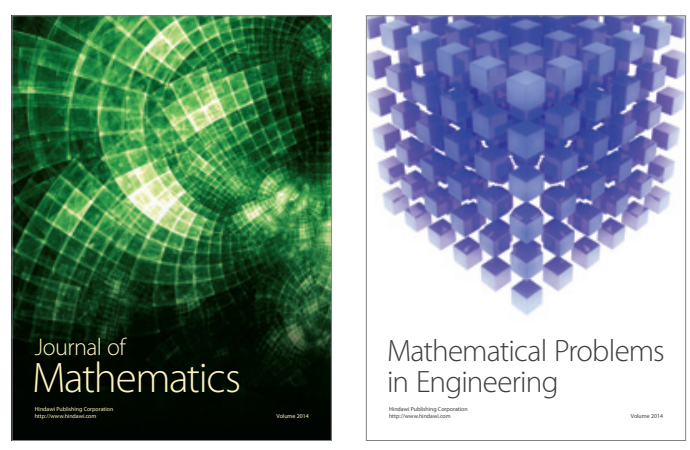

Mathematical Problems in Engineering
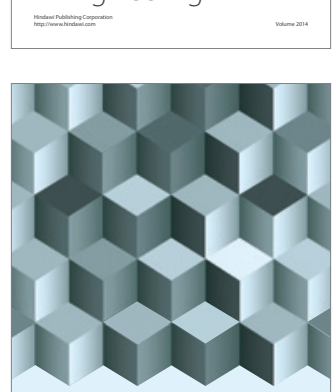

Journal of

Function Spaces
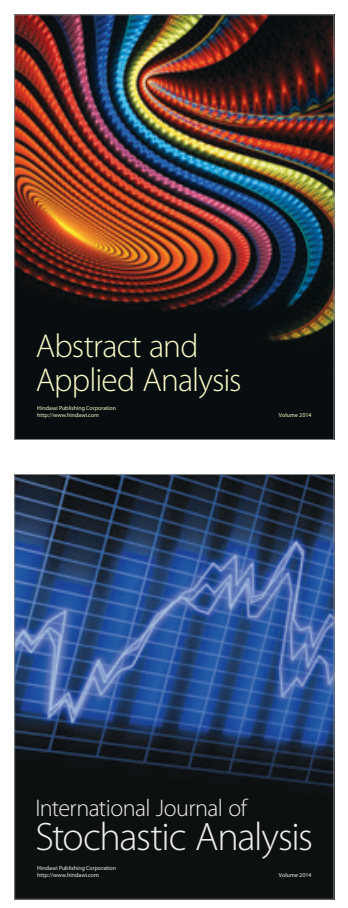

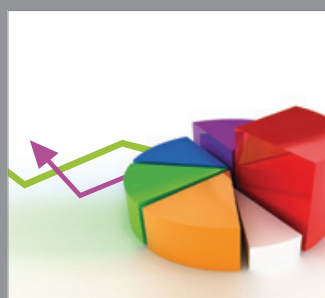

ournal of

Probability and Statistics

Promensencen
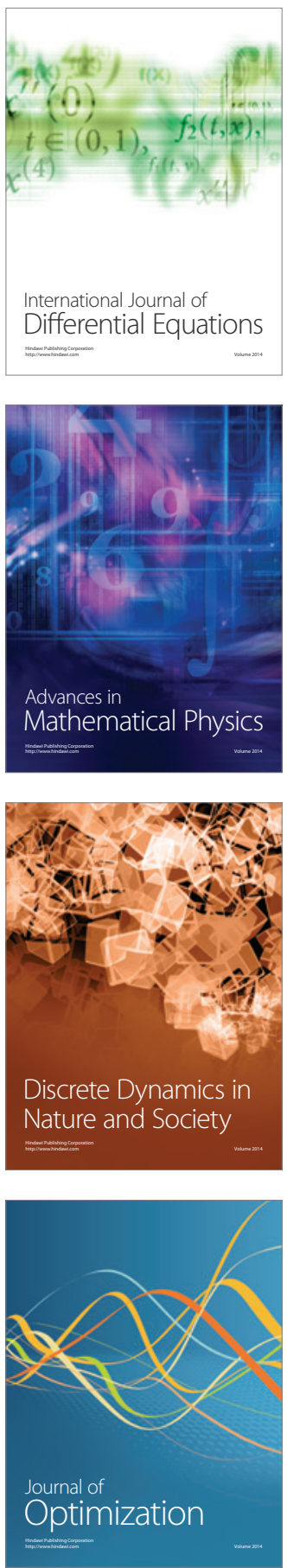